\title{
Modeling Multi-Lane Traffic Flow with Queuing Effects
}

\author{
Dirk Helbing \\ II. Institute of Theoretical Physics, University of Stuttgart, 70550 Stuttgart, Germany
}

\begin{abstract}
On the basis of assumptions about the behavior of driver-vehicle units concerning acceleration, deceleration, overtaking, and lane-changing maneuvers, a gas-kinetic traffic model for uni-directional multi-lane freeways is constructed. Queuing effects are explicitly taken into account in an overall manner. The resulting model is a generalization of Paveri-Fontana's Boltzmann-like traffic model and allows the derivation of macroscopic traffic equations for interacting lanes, including velocity equations. The related effective macroscopic traffic model for the total freeway cross-section is also derived. It provides corrections with respect to previous traffic models, but agrees with them in special cases.
\end{abstract}

Key Words: Multi-lane traffic; Queuing; Gas-kinetic traffic model; Macroscopic traffic equations; Paveri-Fontana model.

PACS numbers: 51.10.+y, 89.40.+k, 47.90.+a, 34.90.+q 


\section{INTRODUCTION}

Apart from microscopic traffic models, in the last decades a number of interrelated macroscopic traffic models have been proposed [1 11]. The motivations for developing these were

- to describe and understand the instabilities of traffic flow [5, 8,11, 13,

- to optimize traffic flow by means of on-line speed-control systems [14 16,

- to make short-term forecasts of traffic volumes for re-routing measures [17 [19],

- to calculate the average travel times, fuel consumption, and vehicle emissions in dependence of traffic volume 4,20 ,

- to predict the effects of additional roads or lanes [20 23].

Most of these models are restricted to uni-directional freeway traffic and treat the different lanes of a road in an overall manner, i.e. like one lane with higher capacity and possibilities for overtaking. However, this kind of simplification is clearly not applicable if there is a disequilibrium between neighboring lanes. Therefore, some researchers carried out empirical investigations of the observed density oscillations between neighboring lanes or proposed models for their mutual influences [24 29].

However, these are phenomenological models which treat inter-lane interactions in a rather heuristic way. Moreover, most of them base on the simple traffic flow model of Lighthill and Whitham which assumes average velocity on each lane to be in equilibrium with density. This assumption is not very well justified, especially for unstable traffic which is characterized by evolving "phantom traffic jams" or stop-and-go waves [5, 8,11,13. It is also questionable for lane mergings or on-ramp traffic where frequently a disequilibrium occurs. However, instabilities or disequilibria may decrease the freeway capacity considerably.

An alternative approach including a phenomenological velocity equation has been proposed by Michalopoulos et al. [29]. It bases on Payne's model [3, [4] which has been severely criticized for several reasons [5, 11, 30 35]. Therefore, we will derive a consistent macroscopic multi-lane model from a gas-kinetic level of description. This is related to Paveri-Fontana's 
Boltzmann-like approach (cf. Sec. II), but explicitly takes into account overtaking and lanechanging maneuvers. An extension to a gas-kinetic traffic model for several driving styles (e.g. aggressive, timid) or vehicle types (e.g. cars, trucks) is easily possible (cf. Sec. ПIQ).

On the way of constructing gas-kinetic traffic equations we face the problem of vehicle queues (platoons) forming at medium and high traffic volumes [36]. Several solutions have been suggested to cope with this. Most of them base on gas-kinetic ideas, but non of them is fully satisfactory, yet. The approach of Andrews 37 40 mainly provides results for stationary traffic situations. Beylich's model 441,42 is so complicated that the corresponding macroscopic traffic equations are not any more derivable or at least not suited for efficient traffic simulations. For this reason, this paper does not distinguish queues of different lengths (like Beylich does) but only freely moving and impeded (queued) vehicles. This approach has some relation to the one of Lampis 43], but it removes its inconsistencies.

The resulting Boltzmann-like model allows a systematic derivation of macroscopic traffic equations not only for the vehicle densities on the different lanes, but also for the associated average velocities (cf. Sec. III). In Sec. IV it is demonstrated how effective macroscopic traffic equations for the total cross-section of a uni-directional road can be obtained from the equations for single lanes. Due to different legal regulations, the traffic dynamics on American freeways is different from that on European ones (which will be called "autobahns" in accordance with Kühne et al. [14,6]) (cf. Sec. II).

A summary and outlook is presented in Sec. V.

\section{BOLTZMANN-LIKE MULTI-LANE THEORY}

The first Boltzmann-like (gas-kinetic) model was proposed by Prigogine and co-workers [44 46]. However, Paveri-Fontana [47 has pointed out that this model has some peculiar properties. Therefore, Paveri-Fontana proposed an improved model that overcomes most of the short-comings of Prigogine's approach. Nevertheless, his model still treats the lanes of a multi-lane road in an overall manner and does not take into account queuing effects. For this reason, an extended Paveri-Fontana-like model will now be constructed.

Let us assume that the motion of an individual vehicle $\alpha$ can be described by several 
variables like its lane $i_{\alpha}(t)$, its place $r_{\alpha}(t)$, its actual velocity $v_{\alpha}(t)$, and its desired velocity $v_{0 \alpha}(t)$ in dependence of time $t$. The phase-space density $\hat{\rho}_{i}\left(r, v, v_{0}, t\right)$ is then determined by the mean number $\Delta n_{i}\left(r, v, v_{0}, t\right)$ of vehicles on lane $i$ that are at a place between $r-\Delta r / 2$ and $r+\Delta r / 2$, driving with a velocity between $v-\Delta v / 2$ and $v+\Delta v / 2$, and having a desired velocity between $v_{0}-\Delta v_{0} / 2$ and $v_{0}+\Delta v_{0} / 2$ at time $t$ :

$$
\begin{aligned}
& \hat{\rho}_{i}\left(r, v, v_{0}, t\right)=\frac{\Delta n_{i}\left(r, v, v_{0}, t\right)}{\Delta r \Delta v \Delta v_{0}} \\
& \quad=\frac{1}{\Delta r \Delta v \Delta v_{0}} \sum_{\alpha} \delta_{i i_{\alpha}(t)}^{r+\Delta r / 2} \int_{r-\Delta r / 2} d r^{\prime} \delta\left(r^{\prime}-r_{\alpha}(t)\right) \int_{v-\Delta v / 2}^{v+\Delta v / 2} d v^{\prime} \delta\left(v^{\prime}-v_{\alpha}(t)\right) \int_{v_{0}-\Delta v_{0} / 2}^{v_{0}+\Delta v_{0} / 2} d v_{0}^{\prime} \delta\left(v_{0}^{\prime}-v_{\alpha}^{0}(t)\right) .
\end{aligned}
$$

Here, $\Delta r, \Delta v$, and $\Delta v_{0}$ are small intervals. $\delta_{i j}$ denotes the Kronecker symbol and $\delta(x-y)$ Dirac's delta function. The notation " $A$ " indicates that a time-dependence only occurs in exceptional cases. Lane numbers $i$ are counted in increasing order from the right-most to the left-most lane, but in Great Britain and Australia the other way round. (For Great Britain and Australia "left" and "right" must always be interchanged.)

The phase-space density $\hat{\rho}_{i}\left(r, v, v_{0}, t\right)$ can be splitted into a term $\hat{\rho}_{i 1}\left(r, v, v_{0}, t\right)$ describing vehicles that can move freely and a term $\hat{\rho}_{i 2}\left(r, v, v_{0}, t\right)$ delineating impeded vehicles that have to move slower than desired since they are queuing behind other vehicles:

$$
\hat{\rho}_{i}\left(r, v, v_{0}, t\right)=\hat{\rho}_{i 1}\left(r, v, v_{0}, t\right)+\hat{\rho}_{i 2}\left(r, v, v_{0}, t\right)
$$

Introducing the proportion $c_{i}\left(r, v, v_{0}, t\right)$ of freely moving vehicles by

$$
c_{i}\left(r, v, v_{0}, t\right) \hat{\rho}_{i}\left(r, v, v_{0}, t\right)=\hat{\rho}_{i 1}\left(r, v, v_{0}, t\right)
$$

we have the relations

$$
0 \leq c_{i}\left(r, v, v_{0}, t\right) \leq 1
$$

and

$$
\hat{\rho}_{i 2}\left(r, v, v_{0}, t\right)=\left[1-c_{i}\left(r, v, v_{0}, t\right)\right] \hat{\rho}_{i}\left(r, v, v_{0}, t\right) .
$$

Now, we utilize the fact that, due to the conservation of the number of vehicles, the phase-space density $\hat{\rho}_{i}\left(r, v, v_{0}, t\right)$ on lane $i$ obeys the continuity equation [20,47,48] 


$$
\begin{aligned}
\frac{\partial \hat{\rho}_{i}}{\partial t}+\frac{\partial}{\partial r}\left(\hat{\rho}_{i} v\right)+\frac{\partial}{\partial v}\left(\hat{\rho}_{i} f_{i}^{0}\right) & =\left(\frac{\partial \hat{\rho}_{i}}{\partial t}\right)_{\mathrm{ad}}+\left(\frac{\partial \hat{\rho}_{i}}{\partial t}\right)_{\mathrm{vd}}+\left(\frac{\partial \hat{\rho}_{i}}{\partial t}\right)_{\mathrm{int}}+\left(\frac{\partial \hat{\rho}_{i}}{\partial t}\right)_{\mathrm{lc}} \\
& +\hat{\nu}_{i}^{+}\left(r, v, v_{0}, t\right)-\hat{\nu}_{i}^{-}\left(r, v, v_{0}, t\right)
\end{aligned}
$$

The second and third term describe temporal changes of the phase-space density $\hat{\rho}_{i}\left(r, v, v_{0}, t\right)$ due to changes $d r / d t=v$ of place $r$ and due to acceleration $f_{i}^{0}$, respectively. We will assume that the proportion $c_{i}\left(r, v, v_{0}, t\right)$ of freely moving vehicles accelerates to their desired velocity $v_{0}$ with a certain relaxation time $T$ so that we have the acceleration law

$$
f_{i}^{0}\left(r, v, v_{0}, t\right)=\frac{c_{i}}{T}\left(v_{0}-v\right)
$$

The terms on the right-hand side of equation (6) reflect changes of phase-space density $\hat{\rho}_{i}\left(r, v, v_{0}, t\right)$ due to discontinuous changes of desired velocity $v_{0}$, actual velocity $v$, or lane $i$. $\nu_{i}^{+}\left(r, v, v_{0}, t\right)$ and $\nu_{i}^{-}\left(r, v, v_{0}, t\right)$ are the rates of vehicles entering and leaving the road at place $r$. They are only different from zero for merging lanes at entrances and exits respectively.

The term

$$
\left(\frac{\partial \hat{\rho}_{i}}{\partial t}\right)_{\mathrm{ad}}=\frac{\tilde{\rho}_{i}(r, v, t)}{T_{\mathrm{r}}}\left[\hat{P}_{0 i}\left(v_{0} ; r, t\right)-P_{0 i}\left(v_{0} ; r, t\right)\right],
$$

where

$$
\tilde{\rho}_{i}(r, v, t)=\int d v_{0} \hat{\rho}_{i}\left(r, v, v_{0}, t\right)
$$

is a reduced phase-space density and $T_{\mathrm{r}} \approx 1 \mathrm{~s}$ is about the reaction time, describes an adaptation of the actual distribution of desired velocities $P_{0 i}\left(v_{0} ; r, t\right)$ to the reasonable distribution of desired velocities $\hat{P}_{0 i}\left(v_{0} ; r, t\right)$ without any related change of actual velocity $v$.

For the reasonable distribution of desired velocities we will assume the functional dependence

$$
\hat{P}_{0 i}\left(v_{0} ; r, \not t\right)=\frac{1}{\sqrt{2 \pi \hat{\theta}_{0 i}}} \mathrm{e}^{-\left[v_{0}-\hat{V}_{0 i}\right]^{2} /\left[2 \hat{\theta}_{0 i}\right]}
$$

which corresponds to a normal distribution and is empirically well justified 49,25,20. The mean value $\hat{V}_{0 i}=\hat{V}_{0 i}(r, \not)$ and variance $\hat{\theta}_{0 i}=\hat{\theta}_{0 i}(r, t)$ of $\hat{P}_{0 i}\left(v_{0} ; r, t\right)$ depend on road conditions and speed limits. Since European autobahns usually do not have speed limits (at least in Germany), $\hat{\theta}_{0 i}$ is larger for these than for American freeways. In addition, on European 
autobahns $\hat{V}_{0 i}$ increases with increasing lane number $i$ since overtaking is only allowed on the left-hand lane.

The term

$$
\left(\frac{\partial \hat{\rho}_{i}}{\partial t}\right)_{\mathrm{vd}}=\frac{\partial^{2}}{\partial v^{2}}\left(\hat{\rho}_{i} \frac{A_{i} v^{2}}{T}\right)
$$

with a dimensionless, density-dependent function $A_{i}$ describes a kind of "velocity diffusion" which takes into account individual fluctuations of velocity due to imperfect driving. For a detailled discussion of this term cf. Refs. [11,20]. It provides contributions to the dynamical variance equations and equations for higher moments, but neither to the density nor to the velocity equations [20].

Before we specify the Boltzmann-like interaction term $\left(\partial \hat{\rho}_{i} / \partial t\right)_{\text {int }}$ and the lane-changing term $\left(\partial \hat{\rho}_{i} / \partial t\right)_{\text {lc }}$ we will discuss some preliminaries. For reasons of simplicity we will only treat vehicle interactions within the same lane as direct pair interactions, i.e. in a Boltzmann-like manner [50]. Lane-changing maneuvers of impeded vehicles that want to escape a queue (i.e. leave and overtake it) may depend on interactions of up to six vehicles (the envisaged vehicle, the vehicle directly in front of it, and up to two vehicles on both neighboring lanes which may prevent overtaking if they are too close). Therefore, we will treat lane-changing maneuvers in an overall manner by specifying overtaking probabilities and waiting times of lane-changing maneuvers (which corresponds to a mean-field approach, cf. Ref. [50]). These probabilities and waiting times dependent on the vehicle densities and maybe also on other quantities.

\section{A. Overtaking}

Let, for example, $P_{i}^{+}$denote the probability that a slower vehicle could be immediately overtaken on the left-hand lane and $P_{i}^{-}$the analogous probability for the right-hand lane. In addition, let $P_{i_{0}} \equiv P_{i_{0}}(r, t)$ be the proportion of driver-vehicle units that desire to move on lane $i_{0}$ (which corresponds to the proportion of vehicles that actually moves on lane $i_{0}$ at small vehicle densities). Then,

$$
p_{i}^{>} \equiv p_{i}^{>}(r, \not t)=\sum_{i_{0}(>i)} P_{i_{0}}(r, \not t)
$$


is the proportion of vehicles on lane $i$ which would prefer to overtake on or change to the left-hand lane $i+1$, whereas

$$
p_{i}^{<} \equiv p_{i}^{<}(r, \not t)=\sum_{i_{0}(<i)} P_{i_{0}}(r, \not t)
$$

is the proportion of vehicles which would prefer to overtake on or change to the right-hand lane $i-1$. For American freeways we will now calculate the probability $p_{i}^{+}\left[p_{i}^{-}\right]$with which vehicles on lane $i$ are immediately overtaken on lane $i+1$ [lane $i-1$ ] by vehicles that also moved on lane $i$ beforehand. Since $P_{i}^{+}\left(1-P_{i}^{-}\right)\left[P_{i}^{-}\left(1-P_{i}^{+}\right)\right]$is the probability that only lane $i+1$ [lane $i-1]$ allows overtaking, and $P_{i}^{+} P_{i}^{-}$is the probability that both neighboring lanes are free, we obtain

$$
\begin{aligned}
p_{i}^{+} & =c_{i}\left\{P_{i}^{+}\left(1-P_{i}^{-}\right)+\left[p_{i}^{>}+\frac{1}{2}\left(1-p_{i}^{>}-p_{i}^{<}\right)\right] P_{i}^{+} P_{i}^{-}\right\} \\
& =c_{i}\left[P_{i}^{+}\left(1-P_{i}^{-}\right)+\frac{1}{2}\left(1+p_{i}^{>}-p_{i}^{<}\right) P_{i}^{+} P_{i}^{-}\right]
\end{aligned}
$$

and

$$
\begin{aligned}
p_{i}^{-} & =c_{i}\left\{P_{i}^{-}\left(1-P_{i}^{+}\right)+\left[p_{i}^{<}+\frac{1}{2}\left(1-p_{i}^{>}-p_{i}^{<}\right)\right] P_{i}^{+} P_{i}^{-}\right\} \\
& =c_{i}\left[P_{i}^{-}\left(1-P_{i}^{+}\right)+\frac{1}{2}\left(1+p_{i}^{<}-p_{i}^{>}\right) P_{i}^{+} P_{i}^{-}\right]
\end{aligned}
$$

for inner lanes. Here, we have taken into account that only the proportion $c_{i}$ of freely moving vehicles is able to immediately overtake. Moreover, we have assumed that driver-vehicle units change towards their desired lane $i_{0}$ if both neighboring lanes allow overtaking. If already being on the desired lane $i_{0}=i$ (which is the case with probability $1-p_{i}^{>}-p_{i}^{<}$), they choose each neighboring lane with probability $1 / 2$. If only one lane is free, this chance is taken irrespective of the desired lane $i_{0}$. From (14) and (15) follows that the total probability of immediate overtaking is

$$
p_{i}^{+}+p_{i}^{-}=c_{i}\left[1-\left(1-P_{i}^{+}\right)\left(1-P_{i}^{-}\right)\right]
$$

Since, on outer lanes, only one lane is available for overtaking maneuvers, we have

$$
p_{i}^{+}=0, \quad p_{i}^{-}=c_{i} P_{i}^{-}
$$

on the left-most lane and 


$$
p_{i}^{-}=0, \quad p_{i}^{+}=c_{i} P_{i}^{+}
$$

on the right-most lane.

Of course, other specifications of $p_{i}^{+}$and $p_{i}^{-}$are also possible. In Europe, for example, overtaking on the right-hand lane is prohibited for free traffic flow (with less than 30 vehicles per kilometer and lane) so that

$$
p_{i}^{+}=c_{i} P_{i}^{+}, \quad p_{i}^{-}=0
$$

on inner lanes and the right-most lane, but

$$
p_{i}^{+}=0, \quad p_{i}^{-}=0
$$

on the left-most lane. However, for congested traffic (with an average velocity less than 80 kilometers per hour) vehicles are also allowed to pass slower vehicles on the right-hand lane so that the situation is similar to American highways, then.

We are now ready to specify the Boltzmann-like interaction term. For not too large vehicle densities it can be written in the form 20

$$
\begin{aligned}
\left(\frac{\partial \hat{\rho}_{i}}{\partial t}\right)_{\mathrm{int}} & =\sum_{i^{\prime}} \int d v^{\prime} \int_{w<v^{\prime}} d w \int d w_{0} W_{2}\left(v, i \mid v^{\prime}, i^{\prime} ; w, i^{\prime}\right) \hat{\rho}_{i^{\prime}}\left(r, v^{\prime}, v_{0}, t\right) \hat{\rho}_{i^{\prime}}\left(r, w, w_{0}, t\right) \\
& -\sum_{i^{\prime}} \int d v^{\prime} \int_{w<v} d w \int d w_{0} W_{2}\left(v^{\prime}, i^{\prime} \mid v, i ; w, i\right) \hat{\rho}_{i}\left(r, v, v_{0}, t\right) \hat{\rho}_{i}\left(r, w, w_{0}, t\right) .
\end{aligned}
$$

Term (21a) describes an increase of phase-space density $\hat{\rho}_{i}\left(r, v, v_{0}, t\right)$ by interactions of a vehicle with actual velocity $v^{\prime}$ and desired velocity $v_{0}$ on line $i^{\prime}$ with a slower vehicle with actual velocity $w<v^{\prime}$ and desired velocity $w_{0}$ causing the former vehicle to change its velocity to $v \neq v^{\prime}$ or its lane to $i \neq i^{\prime}$. The frequency of such interactions is proportional to the phasespace density $\hat{\rho}_{i^{\prime}}\left(r, w, w_{0}, t\right)$ of hindering vehicles and the phase-space density $\hat{\rho}_{i^{\prime}}\left(r, v^{\prime}, v_{0}, t\right)$ of vehicles which can be affected by slower vehicles. Analogously, term (21b) describes a decrease of phase-space density $\hat{\rho}_{i}\left(r, v, v_{0}, t\right)$ by interactions of a vehicle with actual velocity $v$ and desired velocity $v_{0}$ on line $i$ with a slower vehicle with actual velocity $w<v$ and desired velocity $w_{0}$ causing the former vehicle to change its velocity to $v^{\prime} \neq v$ or its lane to $i^{\prime} \neq i$. Since the interaction is assumed not to influence the desired velocities $v_{0}, w_{0}$, the interaction rate $W_{2}$ is independent of these. However, the interaction rate $W_{2}\left(v^{\prime}, i^{\prime} \mid v, i ; w, i\right)$ 
is proportional to the relative velocity $|v-w|$ of approaching vehicles. Therefore, we have the following relation:

$$
\begin{aligned}
W_{2}\left(v^{\prime}, i^{\prime} \mid v, i ; w, i\right) & =p_{i}^{+}|v-w| \delta_{i^{\prime}(i+1)} \delta\left(v^{\prime}-v\right) \\
& +p_{i}^{-}|v-w| \delta_{i^{\prime}(i-1)} \delta\left(v^{\prime}-v\right) \\
& +\left(1-p_{i}\right)|v-w| \delta_{i^{\prime} i} \delta\left(v^{\prime}-w\right)
\end{aligned}
$$

Term (22a) describes an undelayed overtaking on lane $i^{\prime}=i+1$ without any change of velocity $\left(v^{\prime}=v\right)$ by vehicles which would be hindered by slower vehicles on lane $i$. Analogously, term (22b) reflects undelayed overtaking maneuvers on lane $i^{\prime}=i-1$. Term (220) delineates situations where a vehicle cannot be immediately overtaken by a faster vehicle so that the latter must stay on the same lane $\left(i^{\prime}=i\right)$ and decelerate to the velocity $v^{\prime}=w$ of the hindering vehicle.

\section{B. Lane-Changing}

We come now to the specification of the lane-changing term $\left(\partial \hat{\rho}_{i} / \partial t\right)_{\mathrm{lc}}$. This has the form of a master equation:

$$
\begin{aligned}
\left(\frac{\partial \hat{\rho}_{i}}{\partial t}\right)_{\mathrm{lc}} & =\sum_{i^{\prime}(\neq i)} W_{1}\left(i \mid i^{\prime}\right) \hat{\rho}_{i^{\prime}}\left(r, v, v_{0}, t\right) \\
& -\sum_{i^{\prime}(\neq i)} W_{1}\left(i^{\prime} \mid i\right) \hat{\rho}_{i}\left(r, v, v_{0}, t\right)
\end{aligned}
$$

Term (23a) describes an increase of phase-space density $\hat{\rho}_{i}\left(r, v, v_{0}, t\right)$ due to changes from lane $i^{\prime} \neq i$ to lane $i$ by vehicles with actual velocity $v$ and desired velocity $v_{0}$. The frequency of lane-changing maneuvers is proportional to the phase-space density $\hat{\rho}_{i^{\prime}}\left(r, v, v_{0}, t\right)$ of vehicles

which may be interested in lane-changing. Analogously, term (23b) reflects changes from lane $i$ to another lane $i^{\prime}$ causing a decrease of $\hat{\rho}_{i}\left(r, v, v_{0}, t\right)$. For the corresponding rate $W_{1}\left(i^{\prime} \mid i\right)$ of lane-changing maneuvers we assume

$$
\begin{aligned}
W_{1}\left(i^{\prime} \mid i\right) & =\frac{1}{\tilde{T}_{i}^{+}}\left[1-c_{i}\left(r, v, v_{0}, t\right)\right] \delta_{i^{\prime}(i+1)} \\
& +\frac{1}{\tilde{T}_{i}^{-}}\left[1-c_{i}\left(r, v, v_{0}, t\right)\right] \delta_{i^{\prime}(i-1)}
\end{aligned}
$$




$$
\begin{aligned}
& +\frac{p_{i}^{>}}{\hat{T}_{i}^{+}} r_{i} \delta_{i^{\prime}(i+1)} \\
& +\frac{p_{i}^{<}}{\hat{T}_{i}^{-}} r_{i} \delta_{i^{\prime}(i-1)} .
\end{aligned}
$$

Term (24a) describes lane changes by vehicles which are able to escape a queue on the left-hand lane $i^{\prime}=i+1 . \tilde{T}_{i}^{+}$is the average waiting time until this is possible. Analogously, term (24b) reflects delayed overtaking maneuvers by impeded vehicles on the right-hand lane $i^{\prime}=i-1$. For free traffic flow on European autobahns we must set

$$
\frac{1}{\tilde{T}_{i}^{-}}=0
$$

since overtaking is only allowed on the left-hand lane $i^{\prime}=i+1$.

Term (240) delineates spontaneous lane changes to lane $i^{\prime}=i+1$ by vehicles that desire to move on lane $i_{0}>i$. Analogously, (24d) corresponds to changes to lane $i^{\prime}=i-1$ by vehicles that desire to move on lane $i_{0}<i . \hat{T}_{i}^{+}$and $\hat{T}_{i}^{-}$denote the related average waiting times.

For American freeways we have

$$
r_{i} \equiv r_{i}\left(r, v, v_{0}, t\right)=c_{i}\left(r, v, v_{0}, t\right)
$$

since lane changing by queuing vehicles corresponds to delayed overtaking which is described by terms (24a) and (24b). For free traffic flow on European autobahns we have $p_{i}^{>}=0$ due to a regulation prescribing to use the right-most lane if possible. This also applies to queuing vehicles so that, additionally,

$$
r_{i}=1
$$

We close with some remarks regarding the order of magnitude of the waiting times $\tilde{T}_{i}^{ \pm}$ and $\hat{T}_{i}^{ \pm}$. First of all, they depend on the vehicle density on the neighboring lane $i \pm 1$. Moreover, for the left-most lane we must set

$$
\frac{1}{\tilde{T}_{i}^{+}}=0 \quad \text { and } \quad \frac{1}{\hat{T}_{i}^{+}}=0,
$$

whereas for the right-most lane we always have 


$$
\frac{1}{\tilde{T}_{i}^{-}}=0 \quad \text { and } \quad \frac{1}{\hat{T}_{i}^{-}}=0 .
$$

On American freeways, the waiting times $\hat{T}_{i}^{ \pm}$for spontaneous lane changing are greater than the waiting times $\tilde{T}_{i \pm 1}^{\mp}$ for delayed overtaking since overtaking is usually carried out as soon as possible, whereas changing towards the desired lane is done at one's convenience (if one does not intend to leave the next exit), i.e.

$$
\frac{1}{\hat{T}_{i}^{ \pm}}<\frac{1}{\tilde{T}_{i \pm 1}^{\mp}}
$$

On European autobahns, the waiting time $\hat{T}_{i}^{-}$for spontaneous lane changing (to the righthand lane) is even much larger than the waiting time $\tilde{T}_{i-1}^{+}$for delayed overtaking (on the left-hand lane) since the right-hand lane is only accepted when it offers really large gaps. (Nobody wants to move on the slower lane.)

Defining the abbreviations

$$
\frac{1}{T_{i}^{+}}=\frac{1-c_{i}}{\tilde{T}_{i}^{+}}+\frac{p_{i}^{>} r_{i}}{\hat{T}_{i}^{+}}
$$

and

$$
\frac{1}{T_{i}^{-}}=\frac{1-c_{i}}{\tilde{T}_{i}^{-}}+\frac{p_{i}^{<} r_{i}}{\hat{T}_{i}^{-}}
$$

we can rewrite expression (24) in the simple form

$$
W_{1}\left(i^{\prime} \mid i\right)=\frac{1}{T_{i}^{+}} \delta_{i^{\prime}(i+1)}+\frac{1}{T_{i}^{-}} \delta_{i^{\prime}(i-1)} .
$$

\section{Distinction of different vehicle types}

The gas-kinetic traffic model developed above can be easily generalized to cases where effects of different vehicle types (e.g. cars, trucks) or driving styles (e.g. aggressive, timid) are to be investigated. Then, the phase-space density $\hat{\rho}_{i}\left(r, v, v_{0}, t\right)$ can be splitted into partial phase-space densities $\hat{\rho}_{i}^{a}\left(r, v, v_{0}, t\right)$ describing driver-vehicle units of type $a$ :

$$
\hat{\rho}_{i}\left(r, v, v_{0}, t\right)=\sum_{a} \hat{\rho}_{i}^{a}\left(r, v, v_{0}, t\right)
$$


The corresponding gas-kinetic equations have an analogous form to (6) with (7), (8), (11), (21), and (23). They read

$$
\begin{aligned}
\frac{\partial \hat{\rho}_{i}^{a}}{\partial t} & +\frac{\partial}{\partial r}\left(\hat{\rho}_{i}^{a} v\right)+\frac{\partial}{\partial v}\left(\hat{\rho}_{i}^{a} c_{i}^{a} \frac{v_{0}-v}{T^{a}}\right) \\
& =\frac{\tilde{\rho}_{i}^{a}(r, v, t)}{T_{\mathrm{r}}}\left[\hat{P}_{0 i}^{a}\left(v_{0} ; r, t\right)-P_{0 i}^{a}\left(v_{0} ; r, t\right)\right]+\frac{\partial^{2}}{\partial v^{2}}\left(\hat{\rho}_{i}^{a} \frac{A_{i}^{a} v^{2}}{T^{a}}\right) \\
& =\sum_{b} \sum_{i^{\prime}} \int d v^{\prime} \int d w \int d w_{0} W_{2}^{a b}\left(v, i \mid v^{\prime}, i^{\prime} ; w, i^{\prime}\right) \hat{\rho}_{i^{\prime}}^{a}\left(r, v^{\prime}, v_{0}, t\right) \hat{\rho}_{i^{\prime}}^{b}\left(r, w, w_{0}, t\right) \\
& -\sum_{b} \sum_{i^{\prime}} \int d v^{\prime} \int_{w<v} d w \int d w_{0} W_{2}^{a b}\left(v^{\prime}, i^{\prime} \mid v, i ; w, i\right) \hat{\rho}_{i}^{a}\left(r, v, v_{0}, t\right) \hat{\rho}_{i}^{b}\left(r, w, w_{0}, t\right) \\
& +\sum_{i^{\prime}(\neq i)} W_{1}^{a}\left(i \mid i^{\prime}\right) \hat{\rho}_{i^{\prime}}^{a}\left(r, v, v_{0}, t\right)-\sum_{i^{\prime}(\neq i)} W_{1}^{a}\left(i^{\prime} \mid i\right) \hat{\rho}_{i}^{a}\left(r, v, v_{0}, t\right) \\
& +\hat{\nu}_{i}^{a+}\left(r, v, v_{0}, t\right)-\hat{\nu}_{i}^{a-}\left(r, v, v_{0}, t\right) .
\end{aligned}
$$

Here, we have taken into account that the relaxation time $T^{a}$ and the reasonable distribution

of desired velocities $\hat{P}_{0 i}^{a}$ may depend on driving style or vehicle type $a$. The specification of the interaction rates $W_{2}^{a b}$ and the lane-changing rates $W_{1}^{a}$ is analogous to the previous discussion (cf. (22) and (24)).

\section{DERIVATION OF MACROSCOPIC TRAFFIC EQUATIONS}

The gas-kinetic traffic equations are not very suitable for computer simulations since they contain too many variables. Moreover, the phase-space densities are very small quantities and, therefore, subject to considerable fluctuations so that a comparison with empirical data is difficult. However, the special value of gas-kinetic traffic equations is that they allow a systematic derivation of dynamic equations for the macroscopic (collective) quantities one is mainly interested in.

\section{A. Definition of Variables}

The most relevant macroscopic quantities are the vehicle densities

$$
\rho_{i}(r, t)=\int d v \int d v_{0} \hat{\rho}_{i}\left(r, v, v_{0}, t\right)
$$

and the average velocities 


$$
V_{i}(r, t) \equiv\langle v\rangle_{i}=\int d v v P_{i}(v ; r, t)
$$

on lanes $i$. Here, we have applied the notation

$$
F_{i}(r, t) \equiv\left\langle f\left(v, v_{0}\right)\right\rangle_{i}=\int d v \int d v_{0} f\left(v, v_{0}\right) \frac{\hat{\rho}_{i}\left(r, v, v_{0}, t\right)}{\rho_{i}(r, t)}
$$

and introduced the distribution of actual velocities

$$
P_{i}(v ; r, t)=\int d v_{0} \frac{\hat{\rho}_{i}\left(r, v, v_{0}, t\right)}{\rho_{i}(r, t)}=\frac{\tilde{\rho}_{i}(r, v, t)}{\rho_{i}(r, t)}
$$

on lane $i$. Analogous quantities can be defined for vehicles entering and leaving the road at entrances and exits respectively.

$$
\nu_{i}^{ \pm}(r, t)=\int d v \int d v_{0} \nu_{i}^{ \pm}\left(r, v, v_{0}, t\right)
$$

are the rates of entering and leaving vehicles, and

$$
V_{i}^{ \pm}(r, t) \equiv\langle v\rangle_{i}^{ \pm}=\int d v v P_{i}^{ \pm}(v ; r, t)
$$

their average velocities, where

$$
P_{i}^{ \pm}(v ; r, t)=\int d v_{0} \frac{\hat{\nu}_{i}^{ \pm}\left(r, v, v_{0}, t\right)}{\nu_{i}^{ \pm}(r, t)}
$$

are the velocity distributions of entering and leaving vehicles respectively. In addition, we will need the velocity variance

$$
\theta_{i}(r, t) \equiv\left\langle\left[v-V_{i}(r, t)\right]^{2}\right\rangle_{i}=\int d v\left[v-V_{i}(r, t)\right]^{2} P_{i}(v ; r, t)=\left\langle v^{2}\right\rangle_{i}-\left(\langle v\rangle_{i}\right)^{2}
$$

and the average desired velocity

$$
V_{0 i}(r, t)=\int d v \int d v_{0} v_{0} \frac{\hat{\rho}_{i}\left(r, v, v_{0}, t\right)}{\rho_{i}(r, t)}
$$

on each lane $i$ as well as the average interaction rate

$$
\frac{1}{T_{i}^{0}}=\frac{1}{\rho_{i}(r, t)} \int d v \tilde{\rho}_{i}(r, v, t) \int_{w<v} d w(v-w) \tilde{\rho}_{i}(r, w, t)
$$

of a vehicle on lane $i$ with other vehicles on the same lane.

Finally, we will assume that the proportion $c_{i}$ of freely moving vehicles is a function of the density $\rho_{i}$ on the respective lane $i$ : 


$$
c_{i}\left(r, v, v_{0}, t\right) \equiv c_{i}\left[\rho_{i}(r, t)\right] .
$$

The probabilities with which slower vehicles can immediately be overtaken will mainly depend on the density on the neighboring lane $i \pm 1$, i.e.

$$
P_{i}^{ \pm} \equiv P_{i}^{ \pm}\left(\rho_{i \pm 1}\right)
$$

A similar thing holds for the waiting times:

$$
\tilde{T}_{i}^{ \pm} \equiv \tilde{T}_{i}^{ \pm}\left(\rho_{i \pm 1}\right), \quad \hat{T}_{i}^{ \pm} \equiv \hat{T}_{i}^{ \pm}\left(\rho_{i \pm 1}\right) .
$$

Consequently,

$$
p_{i}^{ \pm} \equiv p_{i}^{ \pm}\left(\rho_{i-1}, \rho_{i}, \rho_{i+1} ; r, \not t\right)
$$

and

$$
T_{i}^{ \pm} \equiv T_{i}^{ \pm}\left(\rho_{i}, \rho_{i \pm 1} ; r, t\right) .
$$

From a theoretical standpoint, a generalization to more complicated functional dependences is easily possible, but their determination from empirical data becomes very difficult, then.

\section{B. Derivation of Moment Equations}

We are now ready for deriving the desired macroscopic traffic equations from the gaskinetic equation (6) with (7), (8), (11), (21), (22), (23), and (33). Integration with respect to $v_{0}$ gives us the reduced gas-kinetic traffic equation

$$
\begin{aligned}
\frac{\partial \tilde{\rho}_{i}}{\partial t} & +\frac{\partial}{\partial r}\left(\tilde{\rho}_{i} v\right)+\frac{\partial}{\partial v}\left(\tilde{\rho}_{i} c_{i} \frac{\tilde{V}_{0 i}(v)-v}{T}\right)=\frac{\partial^{2}}{\partial v^{2}}\left(\tilde{\rho}_{i} \frac{A_{i} v^{2}}{T}\right) \\
& -\left(1-p_{i}\right) \tilde{\rho}_{i}(r, v, t) \int d w(v-w) \tilde{\rho}_{i}(r, w, t) \\
& +p_{i-1}^{+} \tilde{\rho}_{i-1}(r, v, t) \int_{w<v} d w(v-w) \tilde{\rho}_{i-1}(r, w, t) \\
& +p_{i+1}^{-} \tilde{\rho}_{i+1}(r, v, t) \int_{w<v} d w(v-w) \tilde{\rho}_{i+1}(r, w, t) \\
& -\left(p_{i}^{+}+p_{i}^{-}\right) \tilde{\rho}_{i}(r, v, t) \int_{w<v} d w(v-w) \tilde{\rho}_{i}(r, w, t)
\end{aligned}
$$




$$
\begin{aligned}
& +\frac{1}{T_{i-1}^{+}} \tilde{\rho}_{i-1}(r, v, t)-\frac{1}{T_{i}^{+}} \tilde{\rho}_{i}(r, v, t) \\
& +\frac{1}{T_{i+1}^{-}} \tilde{\rho}_{i+1}(r, v, t)-\frac{1}{T_{i}^{-}} \tilde{\rho}_{i}(r, v, t) \\
& +\tilde{\nu}_{i}^{+}(r, v, t)-\tilde{\nu}_{i}^{-}(r, v, t)
\end{aligned}
$$

with

$$
\tilde{V}_{0 i}(v) \equiv \tilde{V}_{0 i}(v ; r, t)=\int d v_{0} v_{0} \frac{\hat{\rho}_{i}\left(r, v, v_{0}, t\right)}{\tilde{\rho}_{i}(r, v, t)}
$$

and

$$
\tilde{\nu}_{i}^{ \pm}(r, v, t)=\int d v_{0} \hat{\nu}_{i}^{ \pm}\left(r, v, v_{0}, t\right)
$$

In formula (51), the deceleration term (51b) stems from (22), the terms (51c) to (51d) reflecting immeditate overtaking come from (22a) and (22b), and the lane-changing terms (51f), (51g) originate from (33). The adaptation term $\left(\partial \hat{\rho}_{i} / \partial t\right)_{\text {ad }}$ yields no contribution.

We will now derive equations for the moments $\left\langle v^{k}\right\rangle$ by multiplying (51) with $v^{k}$ and integrating with respect to $v$. Due to

$$
\begin{aligned}
\int d v v^{k} \frac{\partial}{\partial v}\left(\tilde{\rho}_{i} c_{i} \frac{\tilde{V}_{0 i}(v)-v}{T}\right) & =-\int d v k v^{k-1}\left(\tilde{\rho}_{i} c_{i} \frac{\tilde{V}_{0 i}(v)-v}{T}\right) \\
& =-\frac{k c_{i}}{T} \rho_{i}\left(\left\langle v^{k-1} v_{0}\right\rangle_{i}-\left\langle v^{k}\right\rangle_{i}\right), \\
\int d v v^{k} \frac{\partial^{2}}{\partial v^{2}}\left(\tilde{\rho}_{i} \frac{A_{i} v^{2}}{T}\right) & =k(k-1) \frac{\rho_{i} A_{i}}{T}\left\langle v^{k}\right\rangle_{i},
\end{aligned}
$$

and

$$
\left(1-p_{i}\right) \int d v \tilde{\rho}_{i}(r, v, t) \int d w\left(w v^{k}-v^{k+1}\right) \tilde{\rho}_{i}(r, w, t)=\left(1-p_{i}\right)\left(\rho_{i}\right)^{2}\left(\langle v\rangle_{i}\left\langle v^{k}\right\rangle_{i}-\left\langle v^{k+1}\right\rangle_{i}\right)
$$

we obtain the macroscopic moment equations

$$
\begin{aligned}
\frac{\partial}{\partial t}\left(\rho_{i}\left\langle v^{k}\right\rangle_{i}\right)+\frac{\partial}{\partial r}\left(\rho_{i}\left\langle v^{k+1}\right\rangle_{i}\right) & =\frac{k c_{i}}{T} \rho_{i}\left[\left\langle v^{k-1} v_{0}\right\rangle_{i}-\left\langle v^{k}\right\rangle_{i}+(k-1) \frac{A_{i}}{c_{i}}\left\langle v^{k}\right\rangle_{i}\right] \\
& +\left(1-p_{i}\right)\left(\rho_{i}\right)^{2}\left(\langle v\rangle_{i}\left\langle v^{k}\right\rangle_{i}-\left\langle v^{k+1}\right\rangle_{i}\right) \\
& +\frac{p_{i-1}^{+}}{T_{i-1}^{0}} \rho_{i-1}\left[\left\langle v^{k}\right\rangle_{i-1}+\mathcal{K}_{i-1}^{k}\right]-\frac{p_{i}^{+}}{T_{i}^{0}} \rho_{i}\left[\left\langle v^{k}\right\rangle_{i}+\mathcal{K}_{i}^{k}\right] \\
& +\frac{p_{i+1}^{-}}{T_{i+1}^{0}} \rho_{i+1}\left[\left\langle v^{k}\right\rangle_{i+1}+\mathcal{K}_{i+1}^{k}\right]-\frac{p_{i}^{-}}{T_{i}^{0}} \rho_{i}\left[\left\langle v^{k}\right\rangle_{i}+\mathcal{K}_{i}^{k}\right]
\end{aligned}
$$




$$
\begin{aligned}
& +\frac{1}{T_{i-1}^{+}} \rho_{i-1}\left\langle v^{k}\right\rangle_{i-1}-\frac{1}{T_{i}^{+}} \rho_{i}\left\langle v^{k}\right\rangle_{i} \\
& +\frac{1}{T_{i+1}^{-}} \rho_{i+1}\left\langle v^{k}\right\rangle_{i+1}-\frac{1}{T_{i}^{-}} \rho_{i}\left\langle v^{k}\right\rangle_{i} \\
& +\nu_{i}^{+}(r, t)\left\langle v^{k}\right\rangle_{i}^{+}-\nu_{i}^{-}(r, t)\left\langle v^{k}\right\rangle_{i}^{-}
\end{aligned}
$$

where

$$
\left\langle v^{k}\right\rangle_{i}^{ \pm}=\int d v \int d v_{0} v^{k} \frac{\hat{\nu}_{i}^{ \pm}\left(r, v, v_{0}, t\right)}{\nu_{i}^{ \pm}(r, t)}=\int d v v^{k} \frac{\tilde{\nu}_{i}^{ \pm}(r, v, t)}{\nu_{i}^{ \pm}(r, t)}
$$

and

$$
\mathcal{K}_{i}^{k}=\frac{T_{i}^{0}}{\rho_{i}} \int d v \tilde{\rho}_{i}(r, v, t) \int_{w<v} d w v^{k}(v-w) \tilde{\rho}_{i}(r, w, t)-\left\langle v^{k}\right\rangle_{i}
$$

\section{Fluid-Dynamic Multi-Lane Traffic Equations}

In order to derive dynamic equations for the densities $\rho_{i}$ and average velocities $V_{i}$, we need the relations

$$
\left\langle v^{2}\right\rangle_{i}=\left\langle\left[V_{i}+\left(v-V_{i}\right)\right]^{2}\right\rangle_{i}=\left(V_{i}\right)^{2}+2 V_{i}\left\langle v-V_{i}\right\rangle_{i}+\left\langle\left(v-V_{i}\right)^{2}\right\rangle_{i}=\left(V_{i}\right)^{2}+\theta_{i}
$$

and

$$
\rho_{i} \frac{\partial V_{i}}{\partial t}=\frac{\partial}{\partial t}\left(\rho_{i}\langle v\rangle_{i}\right)-V_{i} \frac{\partial \rho_{i}}{\partial t}
$$

Applying these and and using the abbreviations

$$
\frac{1}{\tau_{i}^{ \pm}}=\frac{p_{i}^{ \pm}}{T_{i}^{0}}+\frac{1}{T_{i}^{ \pm}}
$$

equation (57) gives us the density equations

$$
\begin{aligned}
\frac{\partial \rho_{i}}{\partial t}+V_{i} \frac{\partial \rho_{i}}{\partial r} & =-\rho_{i} \frac{\partial V_{i}}{\partial r}+\nu_{i}^{+}(r, t)-\nu_{i}^{-}(r, t) \\
& +\frac{\rho_{i-1}}{\tau_{i-1}^{+}}-\frac{\rho_{i}}{\tau_{i}^{+}}+\frac{\rho_{i+1}}{\tau_{i+1}^{-}}-\frac{\rho_{i}}{\tau_{i}^{-}}
\end{aligned}
$$

This result is similar to previous multi-lane models. In addition, we obtain the velocity equations 


$$
\begin{aligned}
\rho_{i} \frac{\partial V_{i}}{\partial t}+\rho_{i} V_{i} \frac{\partial V_{i}}{\partial r} & =-\frac{\partial\left(\rho_{i} \theta_{i}\right)}{\partial r}+\frac{c_{i} \rho_{i}}{T}\left(V_{0 i}-V_{i}\right)-\left(1-p_{i}\right)\left(\rho_{i}\right)^{2} \theta_{i} \\
& +\frac{\rho_{i-1}}{\tau_{i-1}^{+}}\left(V_{i-1}-V_{i}\right)+\frac{\rho_{i+1}}{\tau_{i+1}^{-}}\left(V_{i+1}-V_{i}\right) \\
& +\frac{p_{i-1}^{+}}{T_{i-1}^{0}} \rho_{i-1} \mathcal{K}_{i-1}^{1}+\frac{p_{i+1}^{-}}{T_{i+1}^{0}} \rho_{i+1} \mathcal{K}_{i+1}^{1}-\frac{p_{i}^{+}+p_{i}^{-}}{T_{i}^{0}} \rho_{i} \mathcal{K}_{i}^{1} \\
& +\nu_{i}^{+}\left(V_{i}^{+}-V_{i}\right)-\nu_{i}^{-}\left(V_{i}^{-}-V_{i}\right)
\end{aligned}
$$

after some lengthy but straightforward calculations. The terms containing the rates $\nu_{i}^{+}$ and $\nu_{i}^{-}$reflect entering and leaving vehicles, respectively. Whereas the terms (63a) and (64a) correspond to the effects of vehicle motion, of acceleration towards the drivers' desired velocities, and of deceleration due to interactions, the terms (63b), (64b), and (64d) arise from overtaking and lane-changing maneuvers. (64b) comes from differences between the average velocities on neighboring lanes. In contrast, $(64 \mathrm{~d})$ originates from the fact that overtaking vehicles are, on average, somewhat faster than the vehicles passed. Whereas (640) tends to produce differences between the average velocities of neighboring lanes, these are reduced by (64b). The term (64d) has a similar form and interpretation like (64b). It is only negligible if entering vehicles are able to adapt to the velocities on the merging lane and exiting vehicles initially have an average velocity similar to that on the lane which they are leaving so that $V_{i}^{ \pm} \approx V_{i}$

In order to close equations (63) and (64), we must specify the interaction rates $1 / T_{i}^{0}$, the correction terms $\mathcal{K}_{i}^{1}$, and the variances $\theta_{i}$. Utilizing that the empirical velocity distributions $P_{i}(v ; r, t)$ are approximately normally distributed [11,25, 49,52, we have

$$
P_{i}(v ; r, t) \approx \frac{1}{\sqrt{2 \pi \theta_{i}(r, t)}} \mathrm{e}^{-\left[v-V_{i}(r, t)\right]^{2} /\left[2 \theta_{i}(r, t)\right]}
$$

which implies

$$
\frac{1}{T_{i}^{0}} \approx \rho_{i} \sqrt{\frac{\theta_{i}}{\pi}}
$$

and

$$
\mathcal{K}_{i}^{1}=\frac{\sqrt{\pi \theta_{i}}}{2}
$$

With a detailled theoretical and empirical analysis it can be shown 20 that the variances $\theta_{i}$ can be well approximated by equilibrium relations of the form 


$$
\theta_{i}=\frac{c_{i}\left(\rho_{i}\right) \mathcal{C}_{i}\left(\rho_{i}, V_{i}\right)+A_{i}\left(\rho_{i}\right)\left(V_{i}\right)^{2}}{c_{i}\left(\rho_{i}\right)-A_{i}\left(\rho_{i}\right)}
$$

where $\mathcal{C}_{i}\left(\rho_{i}, V_{i}\right)=\left\langle\left(v-V_{i}\right)\left(v_{0}-V_{0 i}\right)\right\rangle_{i}^{\mathrm{e}}$ denotes the density- and velocity-dependent equilibrium covariance between actual velocities $v$ and desired velocities $v_{0}$ on lane $i$. In the case of a speed limit there is $v_{0} \approx V_{0 i} \approx V_{0}$ so that $\mathcal{C}_{i} \approx 0$.

For the average desired velocities $V_{0 i}$ we have

$$
V_{0 i} \equiv V_{0 i}(r, t) \approx \hat{V}_{0 i}(r, t)
$$

since

$$
\hat{P}_{0 i}\left(v_{0} ; r, t\right)-P_{0 i}\left(v_{0} ; r, t\right) \approx 0
$$

due to the smallness of $T_{\mathrm{r}}$.

\section{EFFECTIVE MACROSCOPIC EQUATIONS}

We will now investigate how the effective macroscopic traffic equations for the total cross-section of the road look like. For this purpose we introduce the effective (average) density

$$
\rho(r, t)=\frac{1}{I} \sum_{i} \rho_{i}(r, t)
$$

where $I \equiv I(r, t)$ is the number of lanes at place $r$, and calculate the average $\bar{F}_{i}$ over all lanes for every lane-specific quantity $F_{i}$ according to

$$
F \equiv \overline{F_{i}}=\sum_{i} \frac{\rho_{i}}{I \rho} F_{i}
$$

This implies

$$
\rho F=\frac{1}{I} \sum_{i} \rho_{i} F_{i}
$$

In addition we define the effective rates

$$
\nu^{ \pm}(r, t)=\frac{1}{I} \sum_{i} \nu_{i}^{ \pm}(r, t)
$$

of entering and leaving vehicles, respectively, and the corresponding lane-averages 


$$
F^{ \pm} \equiv \overline{F_{i}^{ \pm}}=\sum_{i} \frac{\nu_{i}^{ \pm}}{I \nu^{ \pm}} F_{i}^{ \pm}
$$

In the following, we will apply the factorization approximation

$$
\overline{\rho_{i} F_{i} G_{i}} \approx \overline{\rho_{i} F_{i}} \overline{G_{i}}=\rho F G
$$

Summation of equation (57) over $i$ and division by $I$ gives us

$$
\begin{aligned}
& \frac{\partial}{\partial t}\left(\rho \overline{\left\langle v^{k}\right\rangle_{i}}\right)+\frac{\partial}{\partial r}\left(\rho \overline{\left\langle v^{k+1}\right\rangle_{i}}\right)=\frac{k c}{T} \rho\left[\overline{\left\langle v^{k-1} v_{0}\right\rangle_{i}}-\overline{\left\langle v^{k}\right\rangle_{i}}+(k-1) \overline{\left(A_{i} / c_{i}\right)} \overline{\left\langle v^{k}\right\rangle_{i}}\right] \\
& =(1-p) \rho^{2}\left(\overline{\langle v\rangle_{i}\left\langle v^{k}\right\rangle_{i}}-\overline{\left\langle v^{k+1}\right\rangle_{i}}\right) \\
& +\nu^{+}(r, t) \overline{\left\langle v^{k}\right\rangle_{i}^{+}}-\nu^{-}(r, t) \overline{\left\langle v^{k}\right\rangle_{i}^{-}},
\end{aligned}
$$

since the lane-changing terms cancel out each other:

$$
\frac{1}{I} \sum_{i} \frac{1}{\tau_{i \mp 1}^{ \pm}} \rho_{i \mp 1}\left\langle v^{k}\right\rangle_{i \mp 1}-\frac{1}{I} \sum_{i} \frac{1}{\tau_{i}^{ \pm}} \rho_{i}\left\langle v^{k}\right\rangle_{i}=\frac{1}{I} \sum_{j} \frac{1}{\tau_{j}^{ \pm}} \rho_{j}\left\langle v^{k}\right\rangle_{j}-\frac{1}{I} \sum_{i} \frac{1}{\tau_{i}^{ \pm}} \rho_{i}\left\langle v^{k}\right\rangle_{i}=0 .
$$

A similar thing holds for the terms containing the corrections $\mathcal{K}_{i}^{k}$. As a consequence, only the terms describing vehicle motion, acceleration towards the drivers' desired velocities, velocity fluctuations, deceleration due to vehicle interactions, and entering or leaving vehicles are remaining.

Now, apart from the lane-average $\theta=\overline{\theta_{i}}$ of the velocity variances $\theta_{i}$ we additionally define the total effective variance

$$
\Theta=\overline{\left\langle(v-V)^{2}\right\rangle_{i}}=\overline{\left\langle\left[\left(v-V_{i}\right)+\left(V_{i}-V\right)\right]^{2}\right\rangle_{i}}=\theta+\overline{\left(V_{i}-V\right)^{2}} .
$$

Applying this and relation

$$
\begin{aligned}
\overline{\left\langle v^{2}\right\rangle_{i}} & =\overline{\left(V_{i}\right)^{2}+\theta_{i}}=\overline{\left[V+\left(V_{i}-V\right)\right]^{2}}+\theta \\
& =V^{2}+\overline{\left(V_{i}-V\right)^{2}}+\theta=V^{2}+\Theta
\end{aligned}
$$

to equation (77), after some lengthy but straightforward calculations we obtain the effective density equation

$$
\frac{\partial \rho}{\partial t}+V \frac{\partial \rho}{\partial r}=-\rho \frac{\partial V}{\partial r}+\nu^{+}-\nu^{-}
$$

and the effective velocity equation

$$
\begin{aligned}
\rho \frac{\partial V}{\partial t}+\rho V \frac{\partial V}{\partial r} & =-\frac{\partial(\rho \Theta)}{\partial r}+\frac{c \rho}{T}\left(V_{0}-V\right)-(1-p) \rho^{2} \theta \\
& +\nu^{+}\left(V^{+}-V\right)-\nu^{-}\left(V^{-}-V\right)
\end{aligned}
$$




\section{A. Comparison with former macroscopic traffic models}

Taking a look at the effective density equation (81), we recognize that it has the form of a continuity equation. This reflects the conservation of the number of vehicles, i.e. no vehicle on the considered road is produced or gets lost. The continuity equation was first proposed in the fluid-dynamic traffic model by Lighthill and Whitham [1,51, and it is also a component of most other macroscopic traffic models [3, 5 [11].

The effective velocity equation (82) can alternatively be represented in the form

$$
\rho \frac{\partial V}{\partial t}+\rho V \frac{\partial V}{\partial r}=-\frac{\partial \mathcal{P}}{\partial r}+\frac{\rho}{\tau}\left(V_{\mathrm{e}}-V\right)+\nu^{+}\left(V^{+}-V\right)-\nu^{-}\left(V^{-}-V\right)
$$

where we have introduced the so-called traffic pressure [46,52,53]

$$
\mathcal{P}=\rho \Theta
$$

The effective relaxation time

$$
\tau(\rho)=\frac{T}{c(\rho)}
$$

increases with decreasing effective proportion $c(\rho)$ of freely moving vehicles and reflects the well-known "frustration effect" that the average relaxation time increases with growing vehicle density $\rho$. In addition, we have introduced the equilibrium velocity

$$
V_{\mathrm{e}}=V_{0}-\tau(1-p) \rho \theta
$$

which is determined by the average desired velocity $V_{0}$, diminished by a term arising from deceleration maneuvers due to vehicle interactions. The velocity equations proposed by Prigogine et al. [46], Paveri-Fontana [47], Phillips [52.53], Payne [3], as well as Kerner and Konhäuser [7] can all be written in the form (83) [11]. However, the specification of the functions $\mathcal{P}, \tau$, and $V_{\mathrm{e}}$ is varying from one model to another. The terms $\nu^{ \pm}\left(V^{ \pm}-V\right)$ have been neglected by all previous models, despite the fact that entrances (and exits) reduce the freeway capacity due to $V^{ \pm} \leq V$. Moreover, the effective gas-kinetic models proposed by Prigogine et al. [46], Paveri-Fontana [47, and Phillips [52,53 imply $V_{\mathrm{e}}=V_{0}-\tau(1-p) \rho \Theta$ instead of relation (86). This is a consequence of the fact that they treat the total freeway 
section like a single lane of greater capacity and possibilities for overtaking. However, the approximation $\Theta \approx \theta$ is only valid for $V_{i} \approx V$. Although it is normally justified for American freeways, on European autobahns it is only valid at densities greater than 35 vehicles per kilometer and lane [20].

\section{SUMMARY AND OUTLOOK}

In this paper we have derived a macroscopic traffic model for uni-directional multi-lane roads. Our considerations started from plausible assumptions about the behavior of drivervehicle units regarding acceleration, deceleration, velocity fluctuations, overtaking, and lanechanging maneuvers. In addition, we distinguished freely moving and queuing vehicles since these behave differently. The resulting gas-kinetic traffic model is a generalization of PaveriFontana's Boltzmann-like traffic equation. It can be extended to situations where different vehicle types or driving styles are to be investigated.

The gas-kinetic traffic equations not only allow to derive dynamic equations for the vehicle density on each lane, but also for the average velocity. In addition, we obtained effective macroscopic traffic equations for the total cross-section of the road from the multilane model. The resulting equations implied corrections with respect to previous traffic models, but agree with the ones by Prigogine et al. and Paveri-Fontana in the special case $V_{i} \approx V \approx V^{ \pm}$

Strictly speaking, the presented model is only valid for small vehicle densities. However, with the methods discussed in Refs. [11,20] it can be easily generalized to a traffic model for arbitrary densities. Since the calculations are rather lengthy but straightforward, they have been omitted, here.

Present research focuses on the implementation of the multi-lane model on a computer with the objective of carrying out numerical traffic simulations. The functional relations regarding the probabilities of overtaking, the proportions of freely moving vehicles, the waiting

times for lane-changing maneuvers, etc. are evaluated on the basis of empirical data. Some of the questions we are going to investigate by means of multi-lane simulations are the following: 
1. In which way does on-ramp traffic influence and destabilize the traffic flow on the other lanes? How does the destabilization effect depend on the traffic volume, the length of the on-ramp lane, the total lane number, etc.?

2. In case of a reduction of the number of lanes, is it better to close the left-most or the right-most lane?

3. Is the organization of American freeways or of European autobahns more efficient, or is it a suitable mixture of both? Remember that American freeways are characterized by uniform speed limits and the fact that overtaking as well as lane changing is allowed on both neighboring lanes. In contrast, on European autobahns often no speed limit is prescribed (at least in Germany) and average velocity normally increases with growing lane number since overtaking is only allowed on the left-hand lane.

4. In which traffic situations do stay-in-lane recommendations increase the efficiency of roads? 


\section{REFERENCES}

[1] M. J. Lighthill and G. B. Whitham (1955) Proceedings of the Royal Society A 229, 317.

[2] P. I. Richards (1956) Operations Research 4, 42.

[3] H. J. Payne (1971) In: G. A. Bekey, ed. Mathematical Models of Public Systems, Vol. 1. (Simulation Council, La Jolla, CA).

[4] H. J. Payne (1979) Transportation Research Record 722, 68.

[5] R. D. Kühne (1984) In: I. Volmuller and R. Hamerslag, eds. Proceedings of the 9th International Symposium on Transportation and Traffic Theory. (VNU Science Press, Utrecht, The Netherlands).

[6] R. D. Kühne and M. B. Rödiger (1991) In: B. L. Nelson, W. D. Kelton, and G. M. Clark, eds. Proceedings of the 1991 Winter Simulation Conference. (Society for Computer Simulation International, Phoenix, Arizona).

[7] B. S. Kerner and P. Konhäuser (1993) Physical Review E 48, 2335.

[8] B. S. Kerner and P. Konhäuser (1994) Physical Review E 50, 54.

[9] D. Helbing (1995) Physica A 219, 375.

[10] D. Helbing (1995) Physica A 219, 391.

[11] D. Helbing (1996) Derivation and empirical validation of a refined traffic flow model. Physica A 233, 253.

[12] R. D. Kühne and R. Beckschulte (1993) In: C. F. Daganzo, ed. Proceedings of the 12th International Symposium on the Theory of Traffic Flow and Transportation. (Elsevier, Amsterdam, The Netherlands).

[13] D. Helbing (1995) Physical Review E 51, 3164.

[14] R. D. Kühne (1987) In: N. H. Gartner and N. H. M. Wilson, eds. Proceedings of the 10th International Symposium on Transportation and Traffic Theory. (Elsevier, New York). 
[15] R. D. Kühne and A. Kroen (1992) Knowledge-based optimization of line control systems for freeways. (Steierwald Schönharting \& Partner, Beratende Ingenieure, Stuttgart, Germany).

[16] D. Helbing (1995) In: M. Snorek, M. Sujansky, and A. Verbraeck, eds. Modelling and Simulation 1995. (The Society for Computer Simulation International, Instanbul, Turkey).

[17] M. Hilliges, R. Reiner, and W. Weidlich (1993) In: A. Pave, ed. Modelling and Simulation 1993. (Society for Computer Simulation International, Ghent, Belgium).

[18] M. Hilliges (1995) A phenomenological model for dynamic traffic flow in networks. Transportation Research B 29, 407.

[19] M. Hilliges and N. Koch (1996) In: D. E. Wolf, M. Schreckenberg, and A. Bachem, eds. Traffic and Granular Flow. (World Scientific, Singapore).

[20] D. Helbing Verkehrsdynamik. Neue physikalische Modellierungskonzepte. (Springer, Berlin, 1997).

[21] D. Braess (1968) Unternehmensforschung 12, 258.

[22] T. Bass (May/1992) Road to ruin. Discover, 56.

[23] A. Knop (March/1993) Natur, 76.

[24] D. C. Gazis, R. Herman, and G. H. Weiss (1962) Operations Research 10, 658.

[25] P. K. Munjal and L. A. Pipes (1971) Transportation Research 5, 241.

[26] P. K. Munjal, Y.-S. Hsu, and R. L. Lawrence (1971) Transportation Research 5, 257.

[27] J. Rørbech (1976) Transportation Research Record 596, 22.

[28] Y. Makigami, T. Nakanishi, M. Toyama, and R. Mizote (1983) In: V. F. Hurdle, E. Hauer and G. N. Stewart, eds. Proceedings of the 8th International Symposium on Transportation and Traffic Theory. (University of Toronto Press, Toronto, Ontario). 
[29] P. G. Michalopoulos, D. E. Beskos, and Y. Yamauchi (1984) Transportation Research $B 18,377$.

[30] E. Hauer and V. F. Hurdle (1979) Transportation Research Record 722, 75.

[31] H. J. Payne (1979) In: W. S. Levine, E. Lieberman, and J. J. Fearnsides, eds. Research Directions in Computer Control of Urban Traffic Systems. (American Society of Civil Engineers, New York).

[32] M. Papageorgiou (1983) Applications of Automatic Control Concepts to Traffic Flow Modeling and Control. (Springer, Heidelberg, Germany).

[33] M. Cremer and A. D. May (1985) An Extended Traffic Model for Freeway Control. (Research Report UCB-ITS-RR-85-7. Institute of Transportation Studies, University of California, Berkeley).

[34] S. A. Smulders (1987) In: N. H. Gartner and N. H. M. Wilson, eds. Proceedings of the 10th International Symposium on Transportation and Traffic Theory. (Elsevier, New York).

[35] A. K. Rathi, E. B. Lieberman, and M. Yedlin (1987) Transportation Research Record $1112,61$.

[36] C. F. Daganzo (1975) Transportation Research 9, 339.

[37] F. C. Andrews (1970) Transportation Research 4, 359.

[38] F. C. Andrews (1970) Transportation Research 4, 367.

[39] F. C. Andrews (1973) Transportation Research 7, 223.

[40] F. C. Andrews (1973) Transportation Research 7, 233.

[41] A. E. Beylich (1979) In: R. Campargue, ed. Rarefied Gas Dynamics, Vol. 1. (Commissariat a l'Energie Atomique, Paris, France).

[42] A. E. Beylich (1981) In: G. Adomeit and H.-J. Frieske, eds. Neue Wege in der Mechanik. (VDI-Verlag, Düsseldorf, Germany). 
[43] M. Lampis (1978) Transportation Science 12, 16.

[44] I. Prigogine and F. C. Andrews (1960) Operations Research 8, 789.

[45] I. Prigogine (1961) In: R. Herman, ed. Theory of Traffic Flow. (Elsevier, Amsterdam, The Netherlands).

[46] I. Prigogine and R. Herman (1971) Kinetic Theory of Vehicular Traffic. (Elsevier, New York).

[47] S. L. Paveri-Fontana (1975) Transportation Research 9, 225.

[48] E. Alberti and G. Belli (1978) Transportation Research 12, 33.

[49] F. Pampel (1955) Ein Beitrag zur Berechnung der Leistungsfähigkeit von Straßen. (Kirschbaum, Bielefeld, Germany).

[50] D. Helbing (1995) Quantitative Sociodynamics. Stochastic Methods and Models of Social Interaction Processes. (Kluwer Academic, Dordrecht, The Netherlands).

[51] G. B. Whitham (1974) Linear and Nonlinear Waves. (Wiley, New York).

[52] W. F. Phillips (1977) Kinetic Model for Traffic Flow. (Report No. DOT/RSPD/ DPB/50-77/17. National Technical Information Service, Springfield, VA 22161).

[53] W. F. Phillips (1979) Transportation Planning and Technology 5, 131. 